\title{
BMJ Open Temporal trends in incidence and mortality rates of laryngeal cancer at the global, regional and national levels, 1990-2017
}

\author{
Jing-Yuan Wang (D , ${ }^{1}$ Qiang-Wei Zhang, ${ }^{2}$ Kaixue Wen, ${ }^{1}$ Chen Wang, ${ }^{1}$ Xiaolin Ji, ${ }^{1}$ \\ Lixia Zhang ${ }^{1}$
}

To cite: Wang J-Y, Zhang Q-W, Wen $\mathrm{K}$, et al. Temporal trends in incidence and mortality rates of laryngeal cancer at the global, regional and national levels, 1990-2017. BMJ Open 2021;11:e050387. doi:10.1136/ bmjopen-2021-050387

- Prepublication history for this paper is available online To view these files, please visit the journal online (http://dx.doi org/10.1136/bmjopen-2021050387).

J-YW and Q-WZ contributed equally.

Received 23 February 2021 Accepted 23 September 2021

Check for updates

(c) Author(s) (or their employer(s)) 2021. Re-use permitted under CC BY-NC. No commercial re-use. See rights and permissions. Published by BMJ.

${ }^{1}$ Department of Otolarygology Head and Neck Surgery, Shanxi Bethune Hospital, Shanxi Academy of Medical Sciences, Tongji Shanxi Hospital, Third Hospital of Shanxi Medical University, Taiyuan, Shanxi, People's Republic of China 2Department of

Otorhinolaryngology, Shanxi Provincial People's Hospital Affiliated to Shanxi Medical University, Taiyuan, Shanxi, People's Republic of China

Correspondence to Dr Jing-Yuan Wang; wangjingyuan202009@163.com

\section{ABSTRACT}

Objectives Laryngeal cancer is the most prevalent entity of head and neck cancer. Knowing the trends of incidence and mortality of laryngeal cancer is important for the reduction in related disease burden.

Design Population-based observational study.

Main outcomes and measures The incidence and mortality data of laryngeal cancer were retrieved from the Global Burden of Disease study 2017 online database. The estimated average percentage change was used to quantify the trends of laryngeal cancer incidence and mortality at the global, regional and national levels. Results Globally, the numbers of incident cases and deaths due to laryngeal cancer increased $58.7 \%$ and $33.9 \%$, respectively, from 1990 to 2017 . However, the overall age-standardised incidence rate (ASIR) and agestandardised mortality rate decreased by $0.99 \%$ (95\% Cl $0.83 \%$ to $1.14 \%$ ) and $1.62 \%(95 \% \mathrm{Cl} 1.50 \%$ to $1.74 \%)$ per year, respectively. These decreases were ubiquitous worldwide. However, unfavourable trends in the ASIR of laryngeal cancer were also observed in a total of 51 developing countries.

Conclusions The incidence and mortality rates of laryngeal cancer have significantly decreased at the global level and in most countries over the past three decades. The regions that showed an increasing incidence trend deserve more attention.

\section{INTRODUCTION}

Laryngeal carcinoma is the most common cancer site of the aerodigestive tract and accounts for $25 \%-30 \%$ of all head and neck cancer cases. ${ }^{1}$ In 2018, it was estimated that a total of 177000 cases of laryngeal cancer and 95000 related deaths occurred worldwide. ${ }^{2}$ Laryngeal cancer is diagnosed more commonly in men than in women. ${ }^{2}$ The most well-determined risk factors for laryngeal cancer include smoking and alcohol consumption. ${ }^{34}$ Owing to the disparities in risk factor distribution from country to country, the incidence and mortality patterns of laryngeal cancer are geographically heterogeneous. ${ }^{5} \mathrm{In}$ this context, a comprehensive description of
Strengths and limitations of this study

- We provided the most comprehensive description of laryngeal cancer incidence and mortality.

- The temporal trends of incidence and mortality are critical to update laryngeal cancer prevention strategies.

- The data used in this study lacked individual data.

- Laryngeal cancer cannot be further classified according to the anatomical position.

the epidemiological characteristics of laryngeal cancer at the national level is needed. In addition, the incidence and mortality trends of laryngeal cancer were commonly reported together with those of other head and neck cancers (HNCs) ${ }^{6}$ Few studies have individually described and analysed the epidemiological features of laryngeal cancer in the international/global setting. However, due to the differences in the histology, pathology, clinical manifestation and prognosis of $\mathrm{HNC}$, separately knowing their evolving incidence and mortality rates is important for $\mathrm{HNC}$ prevention.

In the current study, leveraging the data from the 2017 Global Burden of Disease (GBD) study, ${ }^{7}$ we comprehensively described the epidemiology and analysed the temporal trends of incidence and mortality rates of laryngeal cancer at the global, regional and national levels.

\section{METHODS}

Study data

The GBD study provides a tool to quantify health loss from hundreds of diseases, injuries and risk factors so that health systems can be improved and disparities can be eliminated. ${ }^{78}$ GBD data have been widely used to assess the disease burden of cancers. ${ }^{59}$ 
Herein, we retrieved the incidence and mortality data of laryngeal cancer by sex, country or territory, region and single calendar year from 1990 to 2017 using the online Global Health Data Exchange query tool. The electronic searches were complemented with additional epidemiological measures such as 'metric' (ie, 'rate' and 'number'), 'measure' (ie, 'incidence' and 'deaths'), 'year' (ie, from 1990 to 2017), 'sex' (ie, 'male' and 'female'), 'age' (ie, 'all ages' and 'age-standardized') and 'location'. The data were available at four geographical levels: global (level $1)$; five regions in terms of socio-demographic index (SDI; that is, high SDI, high-middle SDI, middle SDI, low-middle SDI and low SDI; (level 2); 21 GBD regions in terms of geography (eg, Western Europe; level 3); and 195 countries or territories (eg, China; level 4). The SDI is a composite indicator of development status that is strongly correlated with health outcomes. It is the geometrical mean of $0-1$ indexes of total fertility rate under the age of 25, mean education for those ages 15 and older and lagdistributed income per capita. As a composite, a location with an SDI of 0 would have a theoretical minimum level of development relevant to health, while a location with an SDI of 1 would have a theoretical maximum level. ${ }^{10}$

The collection and processing procedures of cancer data in GBD studies have been extensively described elsewhere. ${ }^{11}{ }^{12}$ In brief, the crude incidence and mortality data were collected from cancer registries or aggregated databases of the cancer registry, such as 'Cancer Incidence In Five Continents (CI5)'. Data were excluded if they were not representative of the general population (eg, hospital-based registries). Laryngeal cancer was identified by the International Classification of Diseases (ICD)-10 codes C32-C32.9 and the ICD-9 codes 161-161.9 from the cancer databases. Most cancer registries only report cancer incidence. However, if a cancer registry also reported cancer mortality, mortality data were also extracted from the source to be used in the mortality to incidence estimation. For regions that lack incidence data, cancer incidence was estimated from cancer mortality using mortality to incidence ratios. These data were then modelled by cause of death ensemble modelling (CODEm), which is the framework used to model most cause-specific death rates in the GBD study. ${ }^{12}{ }^{13}$ In brief, the CODEm approach is based on the principles that all types of available data should be used even if data quality varies; that individual models but also ensemble models should be tested for their predictive validity; and that the best model or sets of models should be chosen based on the out-of-sample predictive validity. Despite the advanced modelling strategies used in GBD studies, we should bear in mind that the estimates provided were based on modelling data rather than raw data. The national SDIs were also retrieved from the GBD online database.

\section{Statistical analysis}

We used the estimated average percentage change (EAPC) to quantify the trends of laryngeal cancer incidence and mortality from 1990 to $2017{ }^{14}$ The EAPC can be calculated from the regression model, which was fitted to the calendar years with the natural logarithm of the rates (ie, $y=\alpha+\beta x+\varepsilon$, where $y=\ln ($ rate $)$, and $x=$ calendar year). ${ }^{15}$ Pearson correlation tests were applied to assess the correlations between SDIs and other indexes (eg, the incidence and the EAPC of incidence). All statistical analyses were performed using the R program (V.3.6.3, R core team). A p value less than 0.05 was considered statistically significant.

\section{Patient and public involvement}

No patients were involved.

\section{RESULTS}

Incidence and its trend for laryngeal cancer at the global, regional and national levels

Globally, the number of incident cases of laryngeal cancer increased from 132700 in 1990 to 210600 in 2017 (figure 1A; table 1). Approximately $85 \%$ of total cases occurred in men (table 1). The case numbers were highest in regions with high SDIs and decreased with the SDI levels. During the study period, the overall agestandardised incidence rate (ASIR) of laryngeal cancer experienced a significant decrease $(\mathrm{EAPC}=-0.99,95 \%$ CI -1.14 to -0.83 ) (figure 1B). The ASIR decreased with different magnitudes in different regions. The greatest reductions were observed in high-SDI regions (table 1). For GBD regions, the highest ASIR and case number of laryngeal cancer were found in the Caribbean and East Asia, respectively, in 2017 (table 1). Between 1990 and 2017, we found that only 3 of 21 GBD regions, namely, East Asia, the Caribbean and Oceania, experienced significant increases in the ASIR (table 1; figure 1C). In the remaining GBD regions, the ASIR was significantly decreased, with the most pronounced reduction found in Andean Latin America (EAPC $=-2.21,95 \%$ CI -2.41 to -2.00) (table 1; figure 1C). In 2017, the ASIR of laryngeal cancer varied 12-fold worldwide. The highest ASIR was observed in Cuba (8.58/100 000), followed by Seychelles and Montenegro (figure 1D). A total of 51 countries, most of which are located in developing regions, experienced significant increases in the ASIR of laryngeal cancer from 1990 to 2017 (figure 1E). The greatest increase was found in Sri Lanka $(\mathrm{EAPC}=2.31,95 \%$ CI 2.04 to 2.58 ). In contrast, a total of 122 countries experienced significant decreases in the ASIR of laryngeal cancer (figure 1E). The most marked reduction was seen in Bahrain (EAPC $=-4.79,95 \%$ CI -5.37 to -4.21 ).

\section{Mortality and its trend for laryngeal cancer at the global, regional and national levels}

While the number of deaths from laryngeal cancer increased $33.9 \%$ between 1990 and 2017, the corresponding age-standardised mortality rate (ASMR) decreased by $1.62 \%$ per year during the same period (table 1; figure 2A,B). Most deaths occurred in men 

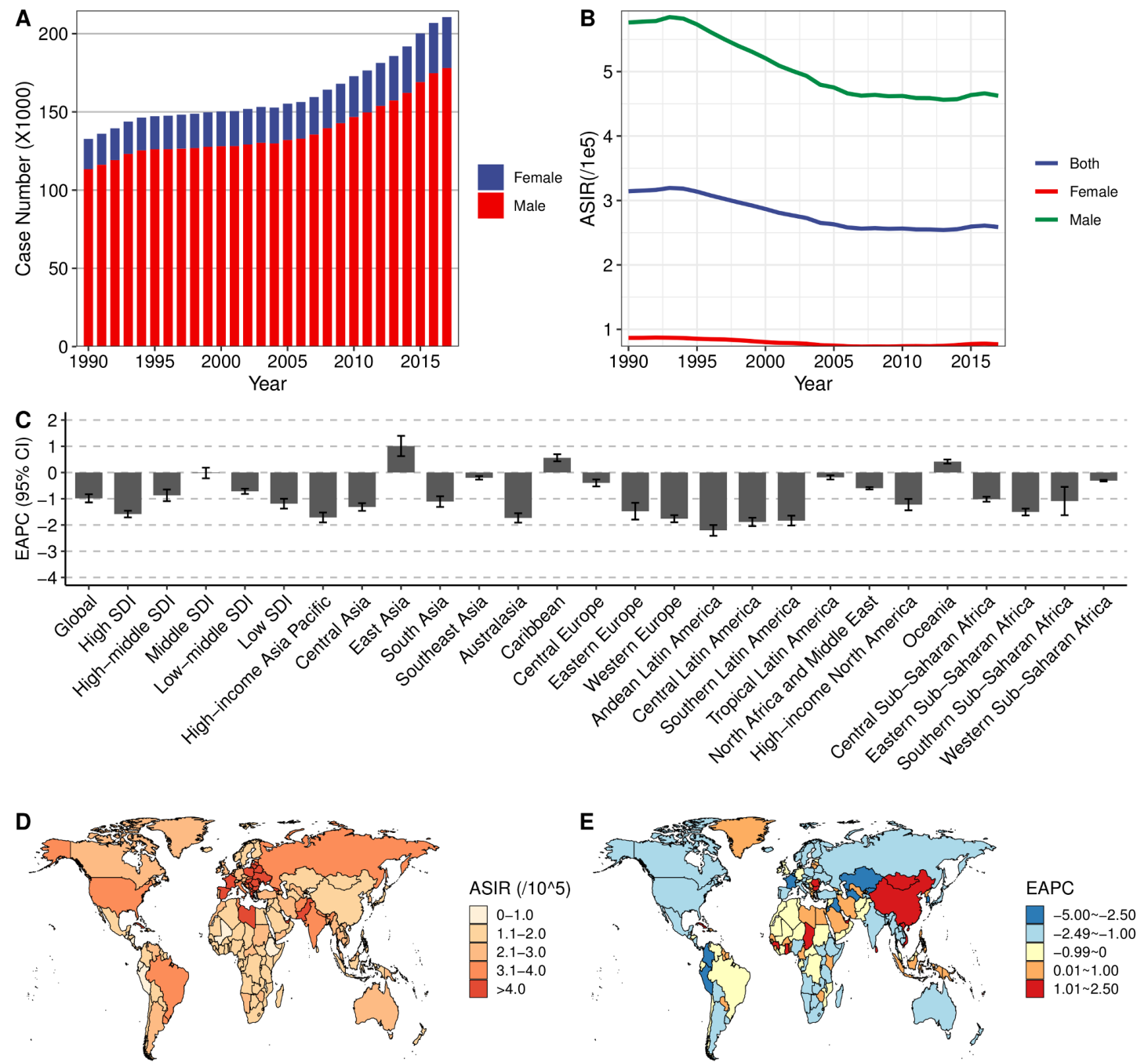

Figure 1 Epidemiological features of laryngeal cancer incidence. (A) The temporal trends of incident case number and (B) agestandardised incidence rate (ASIR) of laryngeal cancer at the global level between 1990 and 2017. (C) The temporal trend of ASIR of laryngeal cancer at the regional level between 1990 and 2017. (D) The ASIR of laryngeal cancer at the national level in 2017. (E) The temporal trend of ASIR of laryngeal cancer at the national level between 1990 and 2017. EAPC, estimated average percentage change; SDI, socio-demographic index.

and in regions with low-to-medium SDIs (table 1). The most significant reductions in the ASMR were found in high-SDI regions. Only one GBD region (ie, Oceania) showed a significant increase in the ASMR of laryngeal cancer between 1990 and 2017. The ASMRs in the remaining GBD regions were significantly decreased (table 1; figure 2C). The greatest decrease was observed in high-income Asia-Pacific (EAPC $=-3.85,95 \% \mathrm{CI},-4.14$ to -3.56). At the national level, the highest ASMR was found in Pakistan $(5.17 / 100$ 000) in 2017, followed by Cuba and Seychelles (figure 2D). Between 1990 and 2017, 30 countries experienced significant increases in the ASMR. The greatest increase was found in Guinea $(\mathrm{EAPC}=1.77$, 95\% CI, 1.56 to 1.98), followed by Chad and Mongolia (figure 2E). In contrast, a total of 150 countries experienced significant decreases in the ASMR of laryngeal cancer. The most marked reduction was found in South Korea $(\mathrm{EAPC}=-7.10,95 \% \mathrm{CI},-7.81$ to -6.40 ) (figure $2 \mathrm{E}$ ).
The associations of SDI with laryngeal cancer-related indexes

We also assessed the associations of the SDI with laryngeal cancer-related indexes at the national level (figure 3). As shown in figure 3A,B, the ASIR and ASMR and their corresponding trends, quantified by the EAPC, of laryngeal cancer were both highly correlated $(\rho=0.795, p<0.001$; $\rho=0.907, p<0.001)$. Countries with a higher SDI had a higher ASIR of laryngeal cancer (figure 3C; $\rho=0.369$, $\mathrm{p}<0.001)$. However, this relationship was reversed for ASMR, although the correlation index was not statistically significant (figure $3 \mathrm{D} ; \rho=-0.127, p=0.079$ ). While no significant association was found between the SDI and the EAPC of ASIR (figure 3E; $\rho=-0.120, p=0.097$ ), a significantly negative association was found between the SDI and the EAPC of ASMR (figure 3F; $\rho=-0.403, p<0.001$ ). These findings suggest that countries with higher SDIs have made greater improvements in the treatment of laryngeal cancer than those with lower SDIs. 

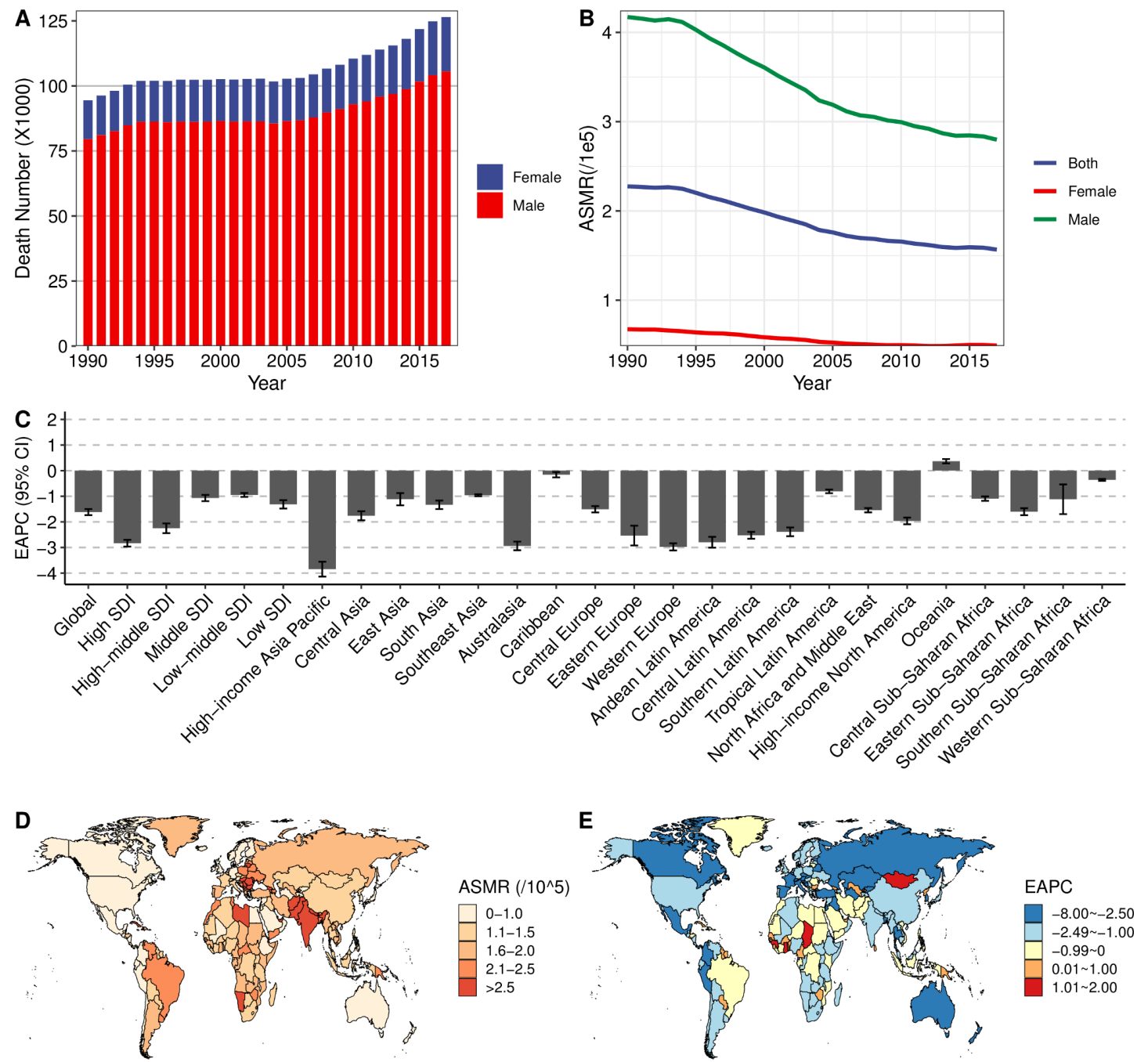

Figure 2 Epidemiological features of laryngeal cancer mortality. (A) The temporal trends of incident death number and (B) agestandardised mortality rate (ASMR) of laryngeal cancer at the global level between 1990 and 2017. (C) The temporal trend of ASMR of laryngeal cancer at the regional level between 1990 and 2017. (D) The ASMR of laryngeal cancer at the national level in 2017. (E) The temporal trend of ASMR of laryngeal cancer at the national level between 1990 and 2017. EAPC, estimated average percentage change; SDI, socio-demographic index.

\section{DISCUSSION}

In this study, we reported the incidence and mortality trends of laryngeal cancer at the global, regional and national levels. In brief, our findings showed high geographical heterogeneity in both the incidence and mortality rates of laryngeal cancer worldwide. We observed significant decreasing trends in the incidence and mortality rates of laryngeal cancer at the global level and in most countries between 1990 and 2017. These reductions suggested the efficacy of current prevention strategies and highlighted the importance of continuous application and even strengthening of these strategies. However, we also observed unfavourable trends in the incidence of laryngeal cancer in a few countries. Most of these countries were located in East Asia and North Africa in areas that lacked advanced economies. Owing to the large population sizes in these countries, the increase in laryngeal cancer incidence might suggest a considerable disease burden of this malignant carcinoma.
Several risk factors have been implicated in the pathogenesis of laryngeal cancer. The most significant of these are tobacco use and alcohol consumption. In addition, the risk of laryngeal cancer increases with the amount of alcohol consumed. ${ }^{16}$ In previous studies conducted in North America, Europe, Japan and Korea, the multivariate relative risks for the highest levels of consumption ranged between 2 and 10 and were 1.94 for $50 \mathrm{~g} /$ day and 3.95 for $100 \mathrm{~g}$ /day in a meta-analysis of 20 studies. ${ }^{17}$ In our study, we found that the incidence of laryngeal cancer was higher in Europe, where alcohol consumption is higher than the global average. ${ }^{18}$ The decreasing trend of laryngeal cancer incidence might be partly attributed to the reduction of alcohol use in these countries. In a recent study conducted in Great Britain, the authors found that for those born post-1985, alcohol abstention rates are increasing and male consumption is decreasing relative to preceding cohorts. ${ }^{19}$ However, in a large-scale modelling study, ${ }^{20}$ the authors reported that between 


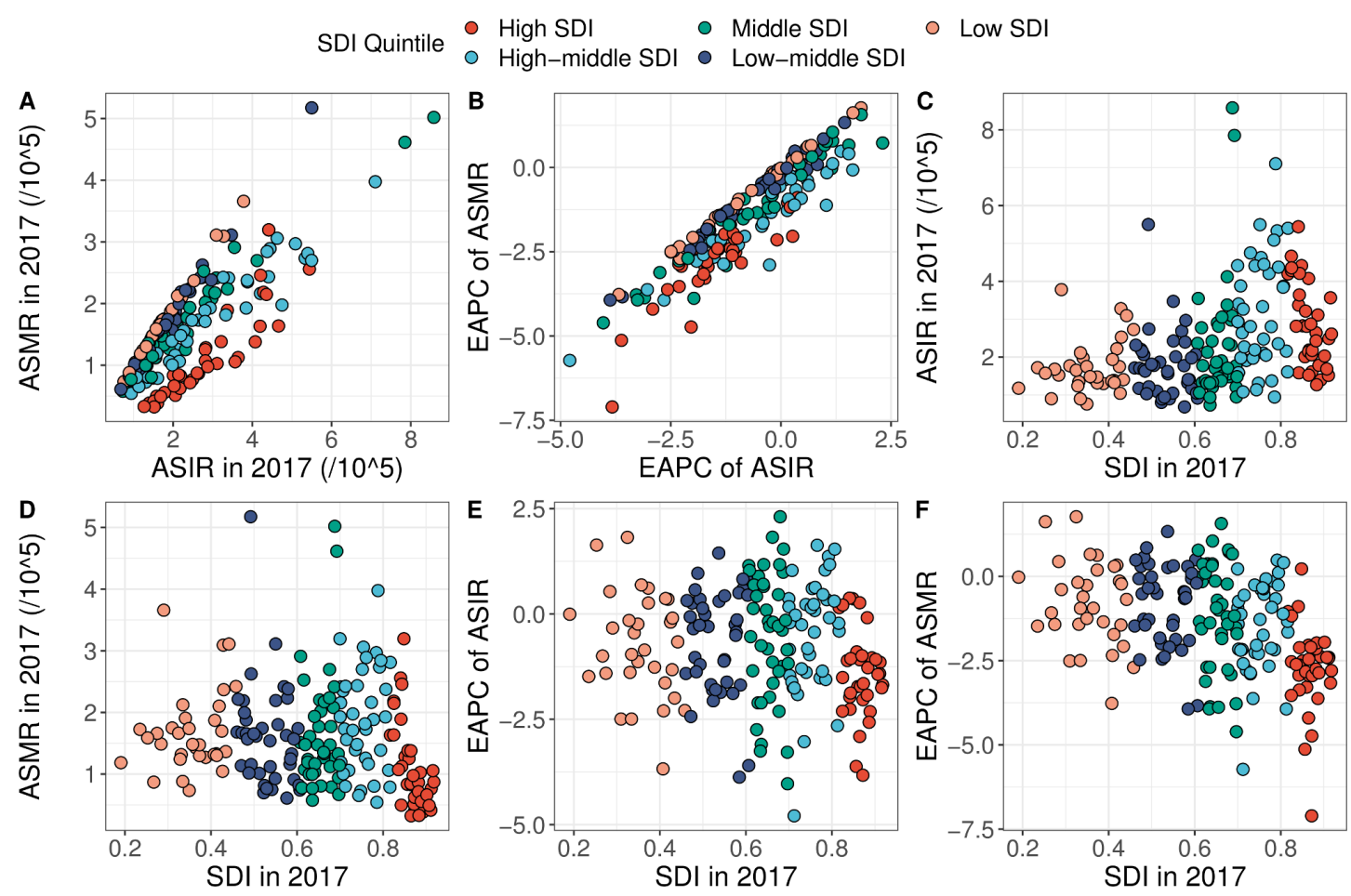

Figure 3 The associations between the national socio-demographic index (SDI) and laryngeal cancer-related indexes. (A) The association between age-standardised incidence rate (ASIR) and age-standardised mortality rate (ASMR) of laryngeal cancer in 2017; (B) the association between changing trends of ASIR and ASMR; (C) the correlations of SDI with ASIR of laryngeal cancer; (D) the correlations of SDI with ASMR of laryngeal cancer; (E) the correlations of SDI with the changing trends of ASIR of laryngeal cancer; $(F)$ the correlations of SDI with the changing trends of ASMR of laryngeal cancer. EAPC, estimated average percentage change.

1990 and 2017, global adult per capita consumption increased from $5.9 \mathrm{~L}$ to $6.5 \mathrm{~L}$ and is forecasted to reach 7.6 L by 2030. In parallel, globally, the prevalence of lifetime abstinence decreased from $46 \%$ in 1990 to $43 \%$ in 2017 and is predicted to further decrease to $40 \%$ in 2030. These results alarmed us that the global goals for reducing the harmful use of alcohol are unlikely to be achieved and that alcohol use-related diseases, including laryngeal cancer, remain the major challenges for human health. In this unfavourable context, although we have seen a significant reduction in the incidence of laryngeal cancer in most countries over the past three decades, the primary preventive strategy of decreasing alcohol use is worth strengthening, such as by increasing the tax of alcohol beverages and strictly forbidding sales to children and adolescents.

Tobacco use has also been shown to have a linear association with the development of laryngeal cancer, with a risk for smokers that is 10-15 times higher than the risk for non-smokers, and the heaviest smokers have as much as a 30 times greater risk. ${ }^{21}$ Fortunately, owing to persistent efforts to combat tobacco use, such as the Framework Convention on Tobacco Control, the prevalence of daily smoking has declined for both men and women in many countries. ${ }^{22}$ However, given the continuing increase in the number of smokers worldwide and the rapid emergence of new tobacco products, additional efforts are needed to achieve a smoke-free world, help smokers quit and protect youth from initiating tobacco use. ${ }^{22}$

Despite the global decrease in the incidence of laryngeal cancer, of note are the unexpected increases in this rate in a few developing countries, such as China and Vietnam. We suspected that these increases might be partly explained by the following reasons: (1) since the improvement of healthcare services and the cancer surveillance system, more laryngeal cancer cases have been diagnosed and recorded; and (2) the prevalence rates of risk factors, especially smoking, are high in these countries. ${ }^{23}{ }^{24}$ For example, the implementation of tobacco control policies in China since the signing of the WHO Framework Convention on Tobacco Control in 2003 has not been effective in reducing smoking prevalence. Smoking prevalence among adolescents of both sexes has increased substantially, and there has been a steady increase in smoking among young women. ${ }^{23}$ A similar phenomenon was observed in Vietnam, in which the reduction in the prevalence of tobacco smoking during the last 5 years was not as high as expected, especially in rural areas. ${ }^{24}$ These findings highlight that more practical and effective policies targeting smoking are urgently needed.

In our study, we also observed a ubiquitous decrease in the mortality rate of laryngeal cancer. Unsurprisingly, the greatest reductions were mostly seen in developed countries. The development of the mortality rate of 
laryngeal cancer was largely based on the evolution of incidence and was likely also due to changes in medical practices, including screening, diagnosis and treatment. ${ }^{1}$ Taking radiotherapy as an example, Atun et al reported that the coverage of radiotherapy was positively associated with gross national income. ${ }^{25}$ Moreover, countries with a relatively higher income will benefit more from investment in radiotherapy than countries with lower or lower-middle income. ${ }^{25}$ Scheduled and opportunistic screening, coupled with efforts to enhance education and health behaviour modification, are highly recommended for predefined, high risk, targeted populations. ${ }^{26}$ These measures can enable early detection and therefore improve mortality. Previous studies have demonstrated the feasibility of developing and implementing large-scale community-based HNC screenings. ${ }^{27}$

The limitations of this study should be noted here. First, all data used in our study were retrieved from GBD studies, which were obtained from mathematical models based on surveillance data rather than the surveillance data itself. Therefore, for countries that lack useable cancer surveillance data, the incidence and mortality of laryngeal cancer are estimated from mathematical models and should be interpreted with caution. In other words, the data used in our study were largely dependent on the quality and quantity of data used in the modelling. For example, the cancer surveillance system was generally more complete in developed countries than in developing countries, which might lead to a larger number of reported cancer cases or a higher ASIR/ASMR of cancer in developed countries. However, GBD studies incorporate sparse data worldwide and provide the most comprehensive data source for the study of cancer disease burden. Second, because of a lag in data availability, estimates for the most recent years are based on past time trends and covariates rather than data, which is reflected in the larger uncertainty. ${ }^{5}$ Finally, we cannot specify laryngeal cancer into several subgroups (eg, glottic carcinoma and supraglottic carcinoma) according to the anatomical position because of data unavailability. A previous study reported that the temporal trends differed markedly between cancers of different anatomical subsites. ${ }^{28}$ Moreover, different subtypes of laryngeal cancer might be considered different entities that are distinct in clinicopathological and immunohistochemical staining characteristics. $^{29}$

In conclusion, we reported global decreases in both the incidence and mortality of laryngeal cancer over the past three decades. These decreases suggest the effectiveness of the control of tobacco and alcohol use. However, unfavourable increases in the incidence of laryngeal cancer were observed in a few developing countries. More targeted and potent prevention strategies for laryngeal cancer are therefore warranted.

Contributors Conceptualisation: J-YW and Q-WZ. Data curation: J-YW, Q-WZ and KW. Formal analysis: J-YW and Q-WZ. Methodology: CW and J-YW. Software: XJ and LZ. Supervision: J-YW and LZ. Roles/Writing —original draft: All authors.
Writing —review and editing: All authors. J-YW is responsible for the overall content as the guarantor.

Funding The authors have not declared a specific grant for this research from any funding agency in the public, commercial or not-for-profit sectors.

Map disclaimer The inclusion of any map (including the depiction of any boundaries therein), or of any geographical or locational reference, does not imply the expression of any opinion whatsoever on the part of BMJ concerning the legal status of any country, territory, jurisdiction or area or of its authorities. Any such expression remains solely that of the relevant source and is not endorsed by BMJ. Maps are provided without any warranty of any kind, either express or implied.

Competing interests None declared.

Patient and public involvement Patients and/or the public were not involved in the design, or conduct, or reporting, or dissemination plans of this research.

Patient consent for publication Not applicable.

Provenance and peer review Not commissioned; externally peer reviewed.

Data availability statement Data are available in a public, open access repository. The data used in our study were available at online Global Health Data Exchange query tool (http://ghdx.healthdata.org/gbd-2017).

Open access This is an open access article distributed in accordance with the Creative Commons Attribution Non Commercial (CC BY-NC 4.0) license, which permits others to distribute, remix, adapt, build upon this work non-commercially, and license their derivative works on different terms, provided the original work is properly cited, appropriate credit is given, any changes made indicated, and the use is non-commercial. See: http://creativecommons.org/licenses/by-nc/4.0/.

ORCID iD

Jing-Yuan Wang http://orcid.org/0000-0001-7633-7302

\section{REFERENCES}

1 Steuer CE, El-Deiry M, Parks JR, et al. An update on larynx cancer. CA Cancer J Clin 2017;67:31-50.

2 Bray F, Ferlay J, Soerjomataram I, et al. Global cancer statistics 2018: GLOBOCAN estimates of incidence and mortality worldwide for 36 cancers in 185 countries. CA Cancer J Clin 2018;68:394-424.

3 Ramroth H, Dietz A, Becher H. Intensity and inhalation of smoking in the aetiology of laryngeal cancer. Int J Environ Res Public Health 2011;8:976-84.

4 La Vecchia C, Zhang ZF, Altieri A. Alcohol and laryngeal cancer: an update. Eur J Cancer Prev 2008;17:116-24.

5 Global Burden of Disease Cancer Collaboration. Global, regional, and National cancer incidence, mortality, years of life lost, years lived with disability, and Disability-Adjusted life-years for 29 cancer groups, 1990 to 2017: a systematic analysis for the global burden of disease study. JAMA Oncol 2019;5:1749-68.

6 Mifsud M, Eskander A, Irish J, et al. Evolving trends in head and neck cancer epidemiology: Ontario, Canada 1993-2010. Head Neck 2017;39:1770-8.

7 Roth GA, Abate D, Abate KH, et al. Global, regional, and national age-sex-specific mortality for 282 causes of death in 195 countries and territories, 1980-2017: a systematic analysis for the global burden of disease study 2017. The Lancet 2018;392:1736-88.

8 GBD 2017 Risk Factor Collaborators. Global, regional, and national comparative risk assessment of 84 behavioural, environmental and occupational, and metabolic risks or clusters of risks for 195 countries and territories, 1990-2017: a systematic analysis for the global burden of disease study 2017. Lancet 2018;392:1923-94.

9 Li N, Deng Y, Zhou L, et al. Global burden of breast cancer and attributable risk factors in 195 countries and territories, from 1990 to 2017: results from the global burden of disease study 2017. J Hematol Oncol 2019;12:140.

10 Global Burden of Disease Collaborative Network. Global burden of disease study 2019 (GBD 2019) socio-demographic index (SDI) 1950-2019. Seattle, United States of America: Institute for Health Metrics and Evaluation (IHME), 2020.

11 GBD 2015 Mortality and Causes of Death Collaborators. Global, regional, and national life expectancy, all-cause mortality, and causespecific mortality for 249 causes of death, 1980-2015: a systematic analysis for the global burden of disease study 2015. Lancet 2016;388:1459-544.

12 GBD 2017 Causes of Death Collaborators. Global, regional, and national age-sex-specific mortality for 282 causes of death in 195 
countries and territories, 1980-2017: a systematic analysis for the global burden of disease study 2017. Lancet 2018;392:1736-88.

13 Foreman KJ, Lozano R, Lopez AD, et al. Modeling causes of death: an integrated approach using CODEm. Popul Health Metr 2012;10:1.

14 Hankey BF, Ries LA, Kosary CL, et al. Partitioning linear trends in age-adjusted rates. Cancer Causes Control 2000;11:31-5.

15 Zheng L, Cui C, Shi O, et al. Incidence and mortality of ovarian cancer at the global, regional, and national levels, 1990-2017. Gynecol Oncol 2020;159:239-47.

16 Altieri A, Garavello W, Bosetti C, et al. Alcohol consumption and risk of laryngeal cancer. Oral Oncol 2005;41:956-65.

17 Bagnardi V, Blangiardo M, La Vecchia C, et al. A meta-analysis of alcohol drinking and cancer risk. Br J Cancer 2001;85:1700-5.

18 Gell L, Meier PS, Goyder E. Alcohol consumption among the over 50S: international comparisons. Alcohol Alcohol 2015;50:1-10.

19 Meng Y, Holmes J, Hill-McManus D, et al. Trend analysis and modelling of gender-specific age, period and birth cohort effects on alcohol abstention and consumption level for drinkers in Great Britain using the general lifestyle survey 1984-2009. Addiction 2014;109:206-15.

20 Manthey J, Shield KD, Rylett M, et al. Global alcohol exposure between 1990 and 2017 and forecasts until 2030: a modelling study. Lancet 2019;393:2493-502.
21 Kuper $\mathrm{H}$, Boffetta $\mathrm{P}$, Adami H-O. Tobacco use and cancer causation: association by tumour type. J Intern Med 2002;252:206-24.

22 Navas-Acien A. Global tobacco use: old and new products. Ann Am Thorac Soc 2018;15:S69-75.

23 Wang M, Luo X, Xu S, et al. Trends in smoking prevalence and implication for chronic diseases in China: serial national cross-sectional surveys from 2003 to 2013. Lancet Respir Med 2019;7:35-45.

24 Van Minh H, Giang KB, Ngoc NB, et al. Prevalence of tobacco smoking in Vietnam: findings from the global adult tobacco survey 2015. Int J Public Health 2017;62:121-9.

25 Atun R, Jaffray DA, Barton MB, et al. Expanding global access to radiotherapy. Lancet Oncol 2015;16:1153-86.

26 Gogarty DS, Shuman A, O'Sullivan EM, et al. Conceiving a national head and neck cancer screening programme. J Laryngol Otol 2016;130:8-14.

27 Hapner ER, Wise JC. Results of a large-scale head and neck cancer screening of an at-risk population. J Voice 2011;25:480-3.

28 Nahavandipour A, Jakobsen KK, Grønhøj C, et al. Incidence and survival of laryngeal cancer in Denmark: a nation-wide study from 1980 to 2014. Acta Oncol 2019;58:977-82.

29 Wachters JE, Kop E, Slagter-Menkema L, et al. Distinct biomarker profiles and clinical characteristics in T1-T2 glottic and supraglottic carcinomas. Laryngoscope 2020;130:2825-32. 